\title{
SIC: AN ADVANCED SEMICONDCTOR MATERIAL FOR POWER DEVICES
}

\author{
Ajay Kumar ${ }^{1}$, M S Aspalli \\ ${ }^{1}$ Dept .Of Electrical \& Electronics Engineering, PDA College of Engineering, Gulbarga - 585 102, Karnataka \\ ${ }^{2}$ Dept .Of Electrical \& Electronics Engineering, PDA College of Engineering, Gulbarga - 585 102, Karnataka
}

\begin{abstract}
Silicon carbide $(\mathrm{SiC})$ is a wide band gap material that shows great promise in high-power and high temperature electronics applications because of its high thermalconductivity and high breakdown electrical field. This paper describes how the outstanding physical andelectronic properties ofSiC permit the fabrication of devices that can operate athigher temperature and power levels than devices fabricated from other material such as silicon orGaAs. In spite of, recent electronics depends primarily upon Silicon based devices; this material is not capable of handling many greater requirements. Devices which operate at high frequency, at high power levels and are to be used in seriousenvironments at high temperatures and high radiation levels required other materialswith wider band gaps than that of silicon. Many space and ground based application rather than satellite applications also have a requirement for wide band gap materials. SiC also has great strength for high power and frequency operation due to a high saturated drift velocity. The Wide band gap permit for unusual optoelectronic applications that include blue light emitting diodes and ultraviolet photo detectors New areas involving gas sensing and RF applications offer significant promise. Overall, the properties of SiC make it one of the best prospects for extending the capabilities and operational regimes of the current semiconductor device technology.
\end{abstract}

Keywords: Silicon carbide, wide band gap semiconductor utility system, power electronics device, physical properties. ***

\section{INTRODUCTION}

Silicon carbide $(\mathrm{SiC})$ is the perfect material between silicon and diamond. The crystal lattice of $\mathrm{SiC}$ is exactly similar to silicon and diamond, but exactly half the lattice sites are filled by silicon atoms and remaining half the lattice sites by carbon atoms. Like-diamond $\mathrm{SiC}$ has electronic properties better properties to silicon.

The wide band gap makes the device operate at high electric fields, and the reduction in intrinsic carrier concentration with increase in band gap enables the device to operate at high temperatures. $\mathrm{SiC}$ is a wide band gap $(3.2 \mathrm{eV})$ Semiconductor with high thermal conductivity, high breakdown electric field strength, high-saturated drift velocity, and high thermal stability. Therefore, silicon carbide is extremely durable and useful for many high power, high frequency, and high temperature applications. The thermal leakage current in $\mathrm{SiC}$ is sixteen orders-of degree lower as well as temperature rises, the escape(leakage) current increases, but the temperature where the leakage current would disturb the circuit operation is over $1000{ }^{\circ} \mathrm{C}$ in $\mathrm{SiC}$, compared to about $250{ }^{\circ} \mathrm{C}$ in silicon. The $\mathrm{SiC}$ electronic period began in the early 1990's whenSiC based single-crystal wafers became commercially available for the first time. During the between years, many different electronic Devices have been demonstrated in $\mathrm{SiC}$ with performance frequently exceeding the theoretical restriction of silicon. These include Pn diodes, MOS field-effect transistors
(MOSFETs),metal-Semiconductor transistors(MESFETs), and bipolar transistors (BJTs.).

\section{CRYSTAL STRUCTURE AND POLYTYPISM}

The SiC's crystalline structure and its polytypic nature influence of polytypism on the physical properties of $\mathrm{SiC}$. Silicon carbide is a binary compound containing equal amount of ' $\mathrm{Si}$ ' and ' $\mathrm{C}$ ', whereSi-C bonds are nearly covalent with an ionic contribution of $12 \%$ ( $\mathrm{Si}$ positively, ' $\mathrm{C}$ ' negatively charged). The smallest building element of any $\mathrm{SiC}$ lattice is a tetrahedron of a $\mathrm{Si}(\mathrm{C})$ atom surrounded by four $\mathrm{C}(\mathrm{Si})$ atoms in strong sp3-bonds. Therefore, the first neighbour shell configuration is identical for all atoms in any crystalline structure of $\mathrm{SiC}$. The basic elements of $\mathrm{SiC}$ crystals are shown in Figure1.

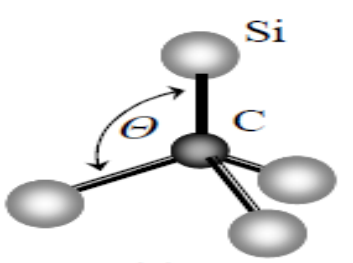

(a)

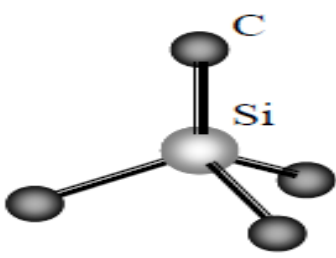

(b)
Fig. 1 Basic elements of $\mathrm{SiC}$ crystals: Tetrahedrons containing (a) one $\mathrm{C}$ and four $\mathrm{Si}$ (b) one $\mathrm{Si}$ and four $\mathrm{C}$ atoms. [1] 


\section{PROPERTIES OF WIDE BAND GAP SEMI- CONDUCTORS}

Table 1 compares the physical properties of $\mathrm{SiC}$ to other importance semiconductor materials such silicon and GaN. The wideband gap material with the most attractive characteristics is diamond. However, a growth process ability of producing mono crystalline diamond in required quantities suitable for semiconductor substrates is not sufficiently available. It is necessary to note that the physical properties stated for $\mathrm{SiC}$ extend into the temperature regime much in excess of $300^{\circ} \mathrm{C}$ which is the almost upper limit of the operational set of conditions for silicon based devices.

Wide band gap materials are less influenced to thermal difficulties and usually have high breakdown electric fields which permit for increased isolation between devices and higher packing densities. Higher thermal conductivities are also of advantage in extent the on-off time (duty cycle) and maximum device packing density. Maximum frequencies/speed at which the devices operated mainly depends on the saturated drift velocity which is clearly an advantage for $\mathrm{SiC}$. In addition, the less dielectric constant for $\mathrm{SiC}$ raises its value for high frequency device operations. Further discussion and comparison among the different semiconductor materials and their properties are in given in Table-1.

Table 1 Comparison of Semiconductor Characteristics [3, 4, 7]

\begin{tabular}{|c|c|c|c|c|}
\hline Pranumber & $\mathbb{8 i}$ & ABS.SC & Gall & Dhanowed \\
\hline Banderangen & 1,11 & 9316 & 339 & 3.47 \\
\hline 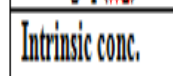 & 1.4M01111 & 89.210 .9 & $\mid 19: 10 \cdot 10$ & $1 \times 14$ \\
\hline 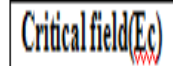 & $M$ & 1.14 & 33 & 3.6 \\
\hline Permithitivite) & 11.1. & 9.7 & 90 & in \\
\hline $\begin{array}{l}\text { Thermal } \\
\text { condurtan (it) }\end{array}$ & 1.5 & 18 & 13 & 10 \\
\hline
\end{tabular}

It is important to mention that the electron mobility and the breakdown electric field are depending on the doping level. The table shows the properties for some standard values.

The SiC substrates can be made in different polytypes i.e. 3C$\mathrm{SiC}, 4 \mathrm{H}-\mathrm{SiC}$ and $6 \mathrm{H}-\mathrm{SiC}$. The ones which are mainly used are $4 \mathrm{H}-\mathrm{SiC}$ and $6 \mathrm{H}-\mathrm{SiC} .4 \mathrm{H}-\mathrm{SiC}$ is best for electronic applications due to its higher carrier mobility and wider band gap in the comparison of other poly types.

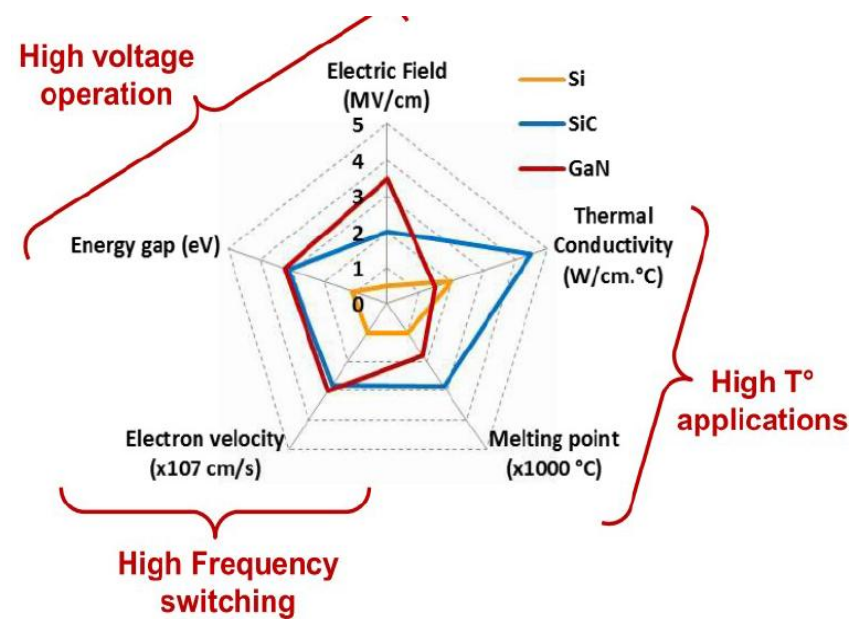

Fig. 2GaN Vs SiC Vs Si of their electrical properties [5]

The intrinsic carrier concentration of $\mathrm{SiC}$ as a function of energy band gap $(\mathrm{Eg})$ is expressed in equation 1 .

$$
n_{i}=\left(\frac{2 \pi k T}{h^{2}}\right)^{\frac{3}{2}}\left(m_{d h} m_{d e}\right)^{\frac{3}{4}} e^{-\frac{E_{g}}{2 k T}} \ldots . . . . .(1)
$$

From equation (1) one can see that the intrinsic carrier density is exponentially decreasing with increasing band gap. This means that $\mathrm{SiC}$ will have a much lower intrinsic carrier density than $\mathrm{Si}$ at a given temperature. The breakdown voltage of a $4 \mathrm{H}$ $\mathrm{SiC}$ junction decreases only by $8 \%$ when the temperature changes from room temperature to $623{ }^{\circ} \mathrm{C}$. It follows from equation (1) that the diffusion current in a Pn-junction is varying with the square of the intrinsic carrier density. Hence, Silicon Carbide Pn-junction has many orders of magnitude lower leakage current than a corresponding Silicon junction at elevated temperatures.

The main contribution to on-state losses in a unipolar device is due to the on-state resistance of the drift region. Thus a good way to compare $\mathrm{SiC}$ and $\mathrm{Si}$ is to compare the specific Resistance R.

Assuming an abrupt, one-dimensional, non punch trough junction fabricated in a uniformly doped semi conductor layer the specific on-resistance is expressed as $[3,4]$

$$
R_{o n, s p}=\frac{4 V_{B}^{2}}{\varepsilon \mu_{n} E_{c}^{3}}
$$

In the above equation $\mathrm{VB}$ is the breakdown voltage $(\mathrm{V}), \mu \mathrm{n}$ is the electron mobility $(\mathrm{cm} / \mathrm{V} \cdot \mathrm{s}), \square$ is the permittivity $(\mathrm{C} / \mathrm{V}-\mathrm{cm})$ and $\mathrm{E}$ stands for the critical electric field $(\mathrm{V} / \mathrm{cm})$. When two devices are compared it is reasonable to choose devices with the 
same breakdown voltage from Equation(2) it is seen that the resistance decrease with increasing electron mobility and critical electrical field. This equation can give an approximation of the ratio between the theoretical resistance in $\mathrm{Si}$ and $\mathrm{SiC}$. Silicon Carbide has one orderof magnitude higher value of the critical field than Silicon, thus the on-state resistance will be much smaller in SiC. To perform a more accurate calculation it is necessary toconsider the mobility's and the breakdown electric fields dependency on the doping level $\mathrm{N}$.

The aim of the critical electric field with a semiconductor device is that they can either block a voltage, or conduct a current with low power loss. As seen in Fig. that the $\mathrm{W}$ is depletion region width at the electric field distribution at inner side of the device.

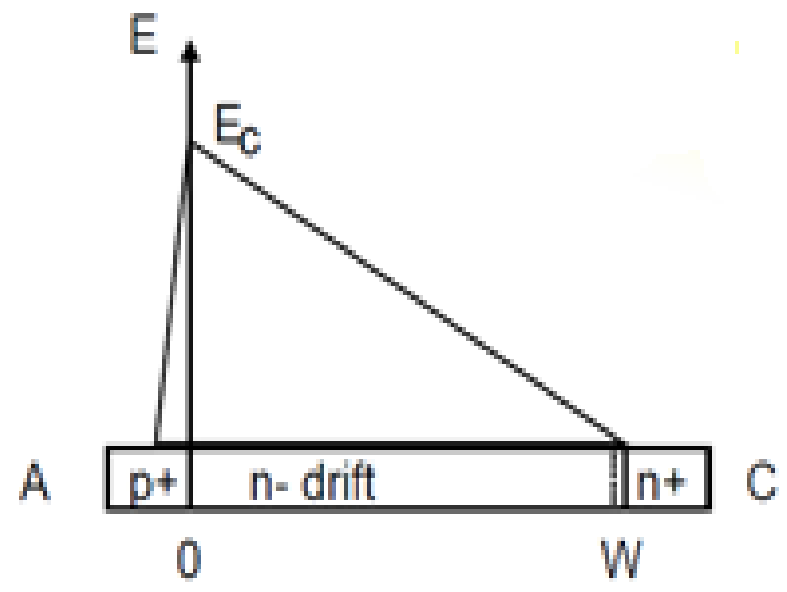

Fig 3Electric field distribution at inner side of the device at Depletion region width [4]

$$
\begin{gathered}
V_{B}=\frac{W E_{c}}{2} \\
W \approx \sqrt{\frac{2 \varepsilon V_{B}}{q N_{D}}} \\
N_{D}=\frac{2 \varepsilon V_{B}}{q W^{2}}=\frac{\varepsilon E_{c}{ }^{2}}{2 q V_{B}} \\
R_{o n, s p}=\frac{W}{q \mu_{N} N_{D}}=\frac{4 V_{B}{ }^{2}}{\varepsilon \mu_{N} E_{c}{ }^{3}} \\
\frac{V_{B}{ }^{2}}{R_{\text {on }, s p}}=\frac{\varepsilon \mu_{N} E_{c}{ }^{3}}{4} \ldots . . .
\end{gathered}
$$

\section{APPLICATIONS OF SIC}

\subsection{Sic - A Widely Used Material}

The material qualities of $\mathrm{SiC}$ have great potential, before the emergence of microelectronics applications based on $\mathrm{SiC}$, to unique mechanical properties, so $\mathrm{SiC}$ is used as an abrasive in sand, in polishing agents or in cutting tools.

$\mathrm{SiC}$ is one of the hardest materials known to man, only diamond and boron nitride are harder. The short bond length of $1.89 \AA$ between ' $\mathrm{Si}$ ' and ' $\mathrm{C}$ ' atoms result is in high bond strength and excellent hardness. However, this makes $\mathrm{SiC}$ wafers difficult to cut and polish. The strong bonds do also create a large band gap that gives $\mathrm{SiC}$ 's high refractive index accompanied by a wide transparency over the visible spectrum, optical intelligence, and resistance to chemical and harsh attack. Recently high purity, almost colourless Moissanite crystals become available, leading to the development of $\mathrm{SiC}$ gemstone that have a beneficial influence on $\mathrm{SiC}$ semiconductor. Due to relatively low density, $\mathrm{SiC}$ can be used even in space applications, e.g. for ultra-lightweight mirrors. It is also appropriate for bearings with the hardness and toughness.

\subsection{Wide Band Gap Semiconductors}

The wide area applications of $\mathrm{SiC}$, is the most promising area is semiconductor processing. The wide band gap materials are better to silicon due to their physical and electrical properties. The properties of different wide band gap semiconductors, selected from the position giving a better view of microelectronics applications. For e.g. in $\mathrm{SiC}$ the probability of thermal excitation of an electron over the band gap is $10^{\wedge}-26$ at room temperature, i.e. there are no thermally excited electrons in the conduction band. The wide band gap is also accompanied by considerably higher breakdown voltage as compared to silicon. This means that for power devices with similar blocking voltage capabilities, the one made of silicon must have about 100 times lower doping level in a 10 times thicker layer, as compared to a $\mathrm{SiC}$ device.

\subsection{SiC for Microelectronics Applications}

The wide band gap semiconductor having silicon dioxide ( $\mathrm{SiO} 2)$ as native oxide, similarly to silicon is studied in $\mathrm{SiC}$. $\mathrm{SiO} 2$ as a dielectric is needed for surface passivation of $\mathrm{SiC}$ devices, as well as for a gate material in metal oxidesemiconductor field-effect transistors (MOSFETs) and related structures. Silicon dioxide can be formed by simple wet or dry oxidation of $\mathrm{SiC}$.

\subsection{High Voltage Devices}

$\mathrm{SiC}$ high voltage devices can be realized on much thinner drift layers then for $\mathrm{Si}$ and $\mathrm{GaAs}$ diodes, succeed high breakdown voltage as well as lower on-resistance and good outcomes have been demonstrated with a fixed improvement in performance. 
$\mathrm{SiC}$ junction Schottky barrier diodes (JBS) are interesting in the $600-3200 \mathrm{~V}$ blocking voltage regime.

\subsection{RF Power Devices}

$\mathrm{SiC}$ devices have shown a very significant improvement and better RF power performance to those available in $\mathrm{Si}$ or $\mathrm{GaAs}$ devices. The RF power available from an impact ionization avalanche transit time (IMPATT) diode in $\mathrm{SiC}$ is $\sim 98$ times higher than in $\mathrm{Si}$. The high temperature and high power $\mathrm{SiC}$ electronic devices would make possible revolutionary improvements to aerospace systems, even without cooling systems. Substitution of hydraulic controls and additional power units with spread over smart electro-mechanicals controls ability of Harsh-surrounding environment (on all sides) operation enables substantial jet-aircraft weight savings, and reduced maintenance.

\subsection{Optoelectronics}

The wide band gap of the different $\mathrm{SiC}$ crystal structures such as $4 \mathrm{H}-\mathrm{SiC}, 6 \mathrm{H}-\mathrm{SIC}$ make them Suitable for far Ultra Violet radiation detection.

High temperature photo detectors are also under development, Ultraviolet photo detectors that are constructed using the different $\mathrm{SiC}$ Crystal structure/poly types in the form of $6 \mathrm{H}-\mathrm{SiC}$ and $4 \mathrm{H}-\mathrm{SiC}$ have been used to recognise UV radiation in the range $200-400 \mathrm{~nm}$. On the other hand Ultraviolet enhanced silicon based photodiodes, by contrast, does not have ability to recognise below $300 \mathrm{~nm}$. The detection peak for a-SiC photodiodes is between 200-300nm, Doping of both U-and $\& \mathrm{SiC}$ can potentially be used to shift the detection peak. SiC operates well up to $600^{\circ} \mathrm{C}-800^{\circ} \mathrm{C}$, while UV detection at these high temperatures is not possible with Silicon Photodiodes because of the low temperature handling capacity of silicon. Dark current ("noise") for $\mathrm{SiC}$ detectors is low; $<10-9 \mathrm{~A} / \mathrm{cn} \sim 2$ at $10 \mathrm{~V}$ and $26^{\circ} \mathrm{C}$, and $10-8 \mathrm{~A} / \mathrm{cn} \sim 2$ at $10 \mathrm{~V}$ and $350^{\circ} \mathrm{C}$ compared to silicon at $10-7 \mathrm{~A} / \mathrm{cn} \sim 2$ at $10 \mathrm{~V}$ and $26^{\circ} \mathrm{C}$. Silicon Carbide is also an indirect band gap material which implies that no $\mathrm{SiC}$ lasers are envisioned. Direct band gap material can easily be achieved, however, from solid solutions ofSiC and two other important materials with wide Gap, AIN and GaN The wide gap range for these materials is about 4 to $6 \mathrm{ev}$ with lower values are achieved by using suitable acceptor and donor impurities. Although $\mathrm{SiC}$ cannot be used as a lasing material, it is a potential waveguide substrate for nitride based optoelectronics. $\mathrm{GaN}$ is a potential wide band gap material for a UV semiconductor laser. The nitride family, however, has proven to be very difficult to grown in a single crystal form. $\mathrm{SiC}$ has been successfully utilized as a substrate for growth of many desirable nitrides with potential usefulness for UV optoelectronics. Relatively high intensity $500 \sim$ m blue LEDs are now commercially available that have been fabricated from $\mathrm{GaN}$ grown on the basal plane of one of the crystal structure $6 \mathrm{H}-\mathrm{SiC}$. In addition, composite structures composed of a $\mathrm{GaN}$ hetero structure grown on an AIN waveguide which in turn has been grown on a supporting substrate of $\mathrm{SiC}$ are expected to provide a variety of promising optoelectronic devices.

\subsection{Sensors}

Semiconductor materials such as Silicon have already shows their great useful properties for pressure sensors such as their large piezoelectric coefficients. The current available operational range of semiconductor pressure sensors is limited up to about $490^{\circ} \mathrm{C}$. There is however a growing demands for sensors capable of operating at temperatures much greater than $490^{\circ} \mathrm{C}$ such as for automotive and avionic down- hole exploration applications. SiC shows their great promising electrical properties as semiconductor material for such potential applications at high temperatures. A pressure sensor that takes advantage of SiC's thermal indifference and hardness could operate in extreme temperature and pressure conditions, such as inside an engine or turbine under the earth.

\section{CONCLUSIONS}

In future with effective effort, wide band gap semiconductors have the favourable time to appropriate the much needed utility requirements to be incorporated power supply where the high temperature, high drift velocity is needed. Among all the wide band gap semiconductor materials diamond has the best electrical properties; research on applying it for high power and high temperature application is only in its preliminary stages, because the processing problem is more unfriendly to solve than any of the other material; however, it likely will be a very significant material for power devices in 20-40 years. In the transition period, there needs to be transition materials with some superior properties. $\mathrm{GaN}$ and $\mathrm{SiC}$ power devices show similarly advantages over Si (Silicon) power devices. GaN's intrinsic properties are slightly better intrinsic properties than $\mathrm{SiC}$; however, there is no availability of pure $\mathrm{GaN}$ wafers, and thus $\mathrm{GaN}$ needs to be grown over $\mathrm{SiC}$ wafer.

$\mathrm{SiC}$ Power devices technology is to a greater extent than GaN technology and is most important in research and commercialization efforts. The small improvement $\mathrm{GaN}$ provides great power not be sufficient to change gears and use $\mathrm{GaN}$ instead of $\mathrm{SiC}$. SiC is the best transition material for future power devices where high temperature and high power is much needed.

\section{REFERENCES}

[1]. T.Paul. Chow, "SiCand GaN High- Voltage Power Switching Devices", Materials Science Forum, vol. 338 -342, Pp 1155-11 60, 2000

[2].Baliga j, "Impact of $\mathrm{SiC}$ on Power Devices", in Amorphous and Crystalline silicon Carbide IV, SpringerVerlag, Barlin Heidelberg, pp.305-313, 1992.

[3]. Leon M. Tolbert, BurakOzpineci, S Kamrul "wide band gap semiconductors for utiltity applications" the University of Tennessee Knoxvile,TN 37996-2100. 
[4]. M .E .Levinshtein ,S.L. Rumyantsev , M.S .Shur, "propertieso advanced semiconductor materials; GaN, GaAa, AIN ,InN , BN ,SiC" ,New York; john wiley and sons, inc20002001.

[5]. Deci-elec "yolo development wide band gap semiconductorin power electronics $17 \& 18$ april 2013.

[6]. A .K. Agarwal, S.S. Mani, S. Seshadri, and J B Cassad, P.A. Sanger and C.D. Brandit, N. Saks, SiCPower Devices," Naval Research Reviews, vol.51, no1. 1999.

[7]. Ned Mohan, T.M Undelend, and W.P .Robbins, PowerElectronics 2 nd edition john willey and sons. 